\title{
Ethnomedicinal Practices for Treating Liver Disorders of Local Communities in the Southern Regions of Korea
}

\author{
Hyun Kim and Mi-Jang Song \\ School of Alternative Medicine and Health Science, Jeonju University, 303 Cheonjam-ro, Wansan-gu, Jeonju 560-759, Republic of Korea \\ Correspondence should be addressed to Hyun Kim; hyunk@jj.ac.kr
}

Received 6 July 2013; Accepted 30 July 2013

Academic Editor: Qing He

Copyright ( $) 2013$ H. Kim and M.-J. Song. This is an open access article distributed under the Creative Commons Attribution License, which permits unrestricted use, distribution, and reproduction in any medium, provided the original work is properly cited.

This study aims to analyze and record ethnomedicinal practices for treating liver disorders of residents living in local communities in the southern regions of Korea. Data was collected using participant observations and in-depth interviews, as the informants also become investigators themselves through attending informal meetings, open and group discussions, and overt observations with semistructured questionnaires. In this study, ethnomedicinal practices for liver ailments were recorded by 1,543 informants (362 men, 1,181 women) at 160 sites. The kinds of liver disorders treated by ethnomedicinal practices were liver cancer, liver cirrhosis, jaundice, hepatitis, fatigue recovery, hangovers, and liver-related ailments. The category with the highest degree of consensus from the informants was jaundice (0.95), and the lowest degree of consensus was for liver cancer and liver cirrhosis (0.61). According to fidelity levels, 28 species resulted in fidelity levels of $100 \%$. The internetwork analysis was first applied for the interpretation of ethnomedicinal knowledge of a community, although it has been strictly used until now for social science in the analysis of social trends and phenomena through the interrelationship of specific components.

\section{Introduction}

After the agreement of the Nagoya Protocol, a stronger interest for sharing the ethnomedicinal knowledge of genetic resources and their benefits to the world has occurred [1]. The ethnomedicinal practices hold significant value among the traditional knowledge of the local communities [2]. Also, ethnomedicinal practices hold an important position in caring for health issues in developing countries [3,4]. Particularly, the ethnomedicinal practices of regions known for traditional medicine include China, Tibet, and India, nations which have actively utilized these treatments for health concerns.

The ethnomedicinal practices of Korea have been continuously successful for over 3,000 years. Korean ethnomedicinal practices contain not only medicinal practice, which include single-medicine proscription, herb fomentation, herb fumigation, and herb ablution, but also nonmedicinal practices like acupuncture, moxibustion, Korean traditional therapeutic touch, Korean traditional saunas, and Korean traditional divination practice. These days, Korean ethnomedicinal practices have been restricted to oral transmission because government policy, after the Korean War, centered on conventional medicine, and the Japanese imperialism, which Korea endured from 1919 to 1945, attempted to annihilate its culture [5].

A scientific investigation of liver disease, the first of its kind, was conducted on indigenous communities in Uttarakhand (India) and was related to jaundice research [6]. In Korea, studies on disease within local communities have been conducted on three separate occasions by the authors: respiratory diseases [7], diseases related to digestion [8], and pain relief [9]; however, this research is the first one to focus strictly on liver disorders.

Our research on liver disorders contains an important meaning to first record and analyze the ethnomedicinal practices of local communities in East Asia, including Korea. Additionally, our research newly included the quantitative analysis method known as internetwork analysis (INA), which has allowed more information to be attained in regard to ethnomedicinal knowledge.

Generally, quantitative analysis for ethnomedicinal knowledge of local communities has solely relied on the 

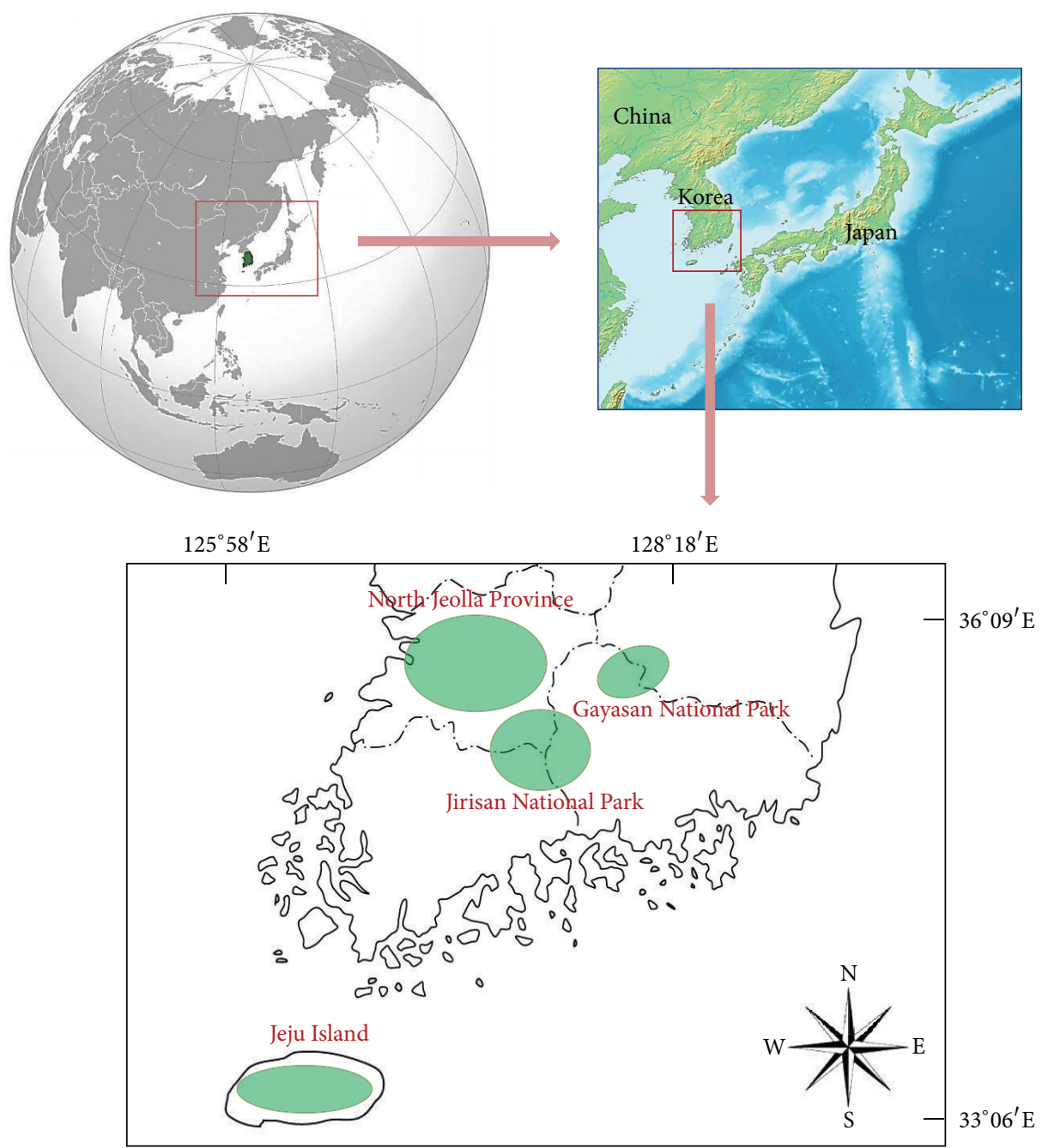

FIGURE 1: Investigation sites.

consensus of the informants and the recorded fidelity levels; therefore, a need exists to utilize the internetwork analysis method to consider the traditional ethnographical properties.

Particularly, a deeper analysis of ethnomedicinal practices in treating specific diseases within the local communities is necessary for obtaining more specific details regarding the internetwork of the components within ethnomedicinal knowledge.

Our research suggests that the applications gained from utilizing the internetwork analysis (INA) for ethnomedicinal practices on liver disorders within communities in Korea will result in further research incorporating INA.

\section{The Research Area and Method}

2.1. Natural and Social Environment of Research Area. The study area consists of the southern region of the Korean peninsula and its many islands, which lie between $33^{\circ} 06^{\prime} \mathrm{N}$ to $36^{\circ} 09^{\prime} \mathrm{N}$ latitude and $125^{\circ} 58^{\prime} \mathrm{E}$ to $128^{\circ} 18^{\prime} \mathrm{E}$ (Figure 1 ). The total population in 2012 of the study area was 5,914,270. The area measures approximately $485 \mathrm{~km}^{2}$ and includes three provinces, 15 cities, and 27 counties in its administrative district [10]. The annual precipitation is around 1,000 1,850 mm in which the coastal area generally receives more rainfall than the inland regions. The annual average temperature of the inland regions is $13.8^{\circ} \mathrm{C}$ while Jeju Island records $16.2^{\circ} \mathrm{C}$ [11].

2.2. Research Methods. Field investigations were conducted from March 2009 to November 2012. Proper data was collected using participant observations and in-depth interviews, as the informants also become investigators themselves through attending informal meetings, open and group discussions, and overt observations with semistructured questionnaires $[12,13]$.

The content of the semi-structured questionnaires was composed of diverse information regarding medicinal species used to treat liver disorders, including local names, used parts, ailments, methods of preparation, manufacturing and administration, dosage, and the usable duration regarding each curable formula [13-15].

All specimens were collected during their flowering or fruiting seasons and were organized utilizing the normal specimen manufacturing method $[15,16]$. The voucher 
specimens were deposited for preservation in the herbarium of Jeonju University. The precise identification of species mentioned by the informants was performed in accordance with Lee [17], Lee [18], Ahn [19], Lee [20], and Park [21]. Scientific names were confirmed by the National Knowledge and Information System for Biological Species [22] of Korea.

\subsection{Quantitative Analysis}

2.3.1. Informant Consensus Factor (ICF). The ICF was used to analyze the agreement degree of the informants' knowledge about each category of ailments [23, 24]. The ICF was calculated using the following formula:

$$
\mathrm{ICF}=\frac{\left(n_{\mathrm{ur}}-n_{t}\right)}{\left(n_{\mathrm{ur}}-1\right)},
$$

where $n_{\text {ur }}$ is the number of use reports of informants for a particular liver disorder and $n_{t}$ is the number of species used by all informants for a particular liver disorder.

2.3.2. Fidelity Level (FL). The FL was employed to determine the most important species used for treating certain liver disorders by the local practitioners and the elderly people living in the study area $[13,16,25]$. The FL was calculated using the following formula:

$$
\mathrm{FL}(\%)=N_{p} \times \frac{100}{N}
$$

where $N_{p}$ is the number of informants that mentioned the specific species used to treat certain disorders and $N$ is the total number of the informants who utilized the species as medicine for treating any given disorder.

2.3.3. Internetwork Analysis (INA). Internetwork analysis does not focus on the independent characteristics of an individual within the community, but it considers the results of the interrelationship among each individual of a community. Internetwork analysis has been applied within communities to various ethnographical problems, including ethnogenesis [26] and obesity [27-29]; however, prior to this research, the internetwork analysis had yet to be applied to ethnomedicinal knowledge, included with its ethnographical properties in the results.

Our research newly applied this method in order to attain more internetwork information from the treatment of ethnomedicinal practices on liver disorders within communities in Korea. The results of the internetwork analysis of disorders and medicinal species were analyzed using UCINET (Ver. 6.460) and NetDraw (Ver. 2.125) software [30, 31].

\section{Results and Discussion}

3.1. Demographic Characteristics of the Region. All 2,069 informants were randomly selected at the community halls, the senior welfare centers, and the traditional markets at 305 sites. Among them, ethnomedicinal practices for liver disorders were recorded by 1,543 informants (362 men,
TABLE 1: Demographic characteristics.

\begin{tabular}{lc}
\hline Gender & \\
Male & $362(23.5 \%)$ \\
Female & $1,181(76.5 \%)$ \\
Age & \\
$30-39$ & $9(0.6 \%)$ \\
$40-49$ & $4(0.3 \%)$ \\
$50-59$ & $73(4.7 \%)$ \\
$60-69$ & $250(16.2 \%)$ \\
$70-79$ & $707(45.8 \%)$ \\
$80-89$ & $460(29.8 \%)$ \\
$90-99$ & $40(2.6 \%)$ \\
Educational attainment & \\
Never attended school & $1,127(73.0 \%)$ \\
Attended school for less than 6 years & $125(8.1 \%)$ \\
Attended school for 6 years & $133(8.6 \%)$ \\
Finished middle school & $101(6.5 \%)$ \\
Finished high school & $57(3.7 \%)$ \\
\hline
\end{tabular}

1,181 women) at 160 sites (Figure 1). The average age of the informants was 75 years old, with a range in age from 36 to 94 , with residents living more than 40 years in the study area. The ethnographical characteristics of the communities are summarized in Table 1.

Linguistically, the inland communities represented varying properties between the eastern and western communities, the Jirisan axis $(1,915 \mathrm{~m})$. The pronunciation of the two local communities depicts dissimilar intonations, while the languages of the communities on Jeju Island possess numerous dialects different from the inland communities.

In regard to foods, the local communities in the eastern region widely used the seed powder of Zanthoxylum piperitum (L.) DC. and the leaves of the Isodon japonicus (Burm.) Hara, while local communities in the western region did not consume these foods. Also, the food traditions in communities on Jeju Island are quite diverse from foods of the inland communities in regard to the recipe and ingredients.

The local communities in the east are politically conservative, while local inland communities in the west are more progressive. However, the communities on Jeju Island display extreme exclusiveness because of their historical experiences.

In homes within the inland communities, men usually support their families financially, while women traditionally support their families on Jeju Island.

3.2. Analysis of Ethnomedicinal Practices. The kinds of liver disorders treated by ethnomedicinal practices were liver cancer, liver cirrhosis, jaundice, hepatitis, fatigue recovery, hangovers, and liver-related ailments (Table 2). Also, this study area is three times larger than previous research, while the seven types of liver disorders recorded in this study were less than previous research, which classified 14 types of respiratory system diseases, 29 types of digestive system diseases, and 23 types of pain relief treatment [7-9]. We believe that the communities of this study area possess 


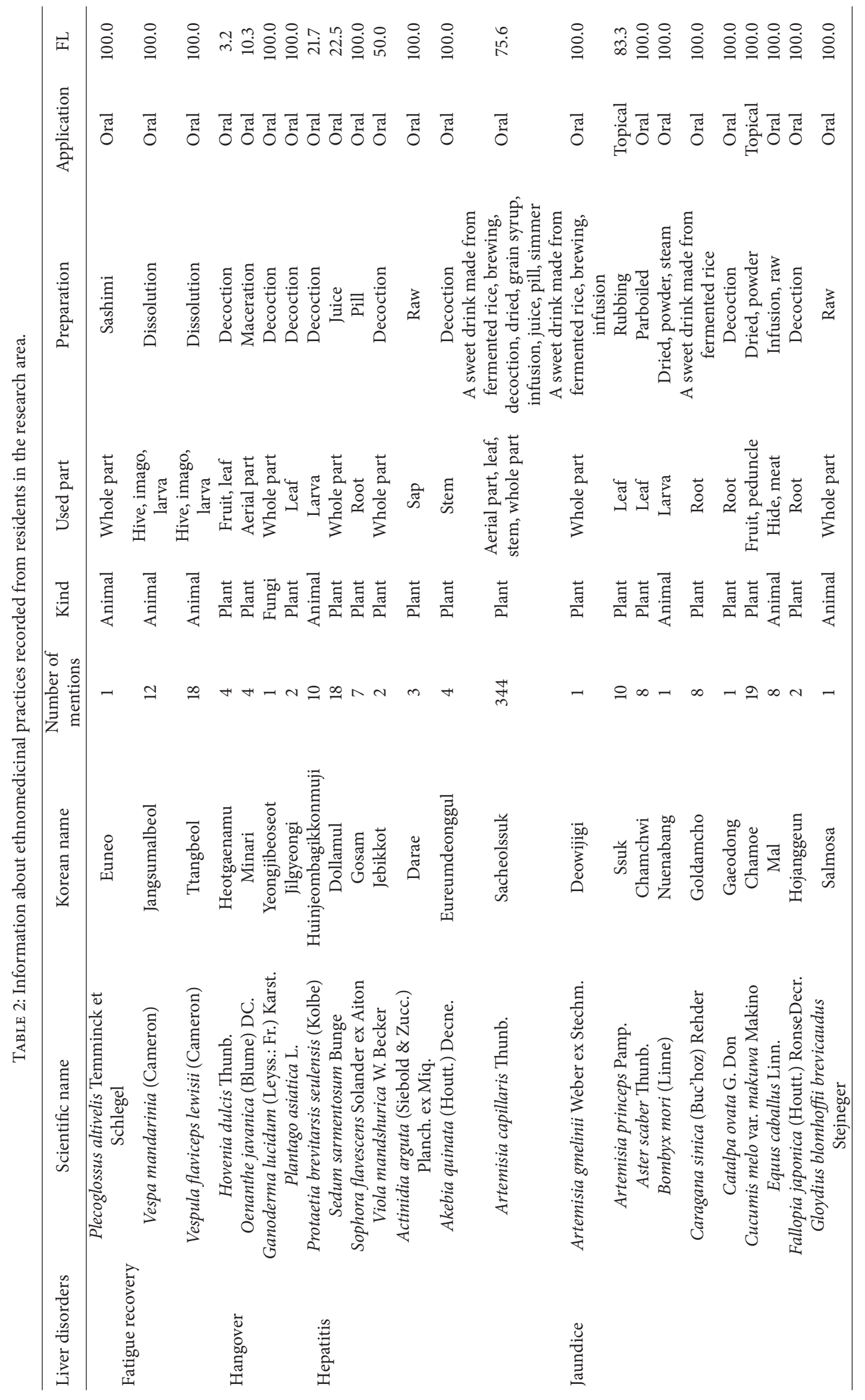




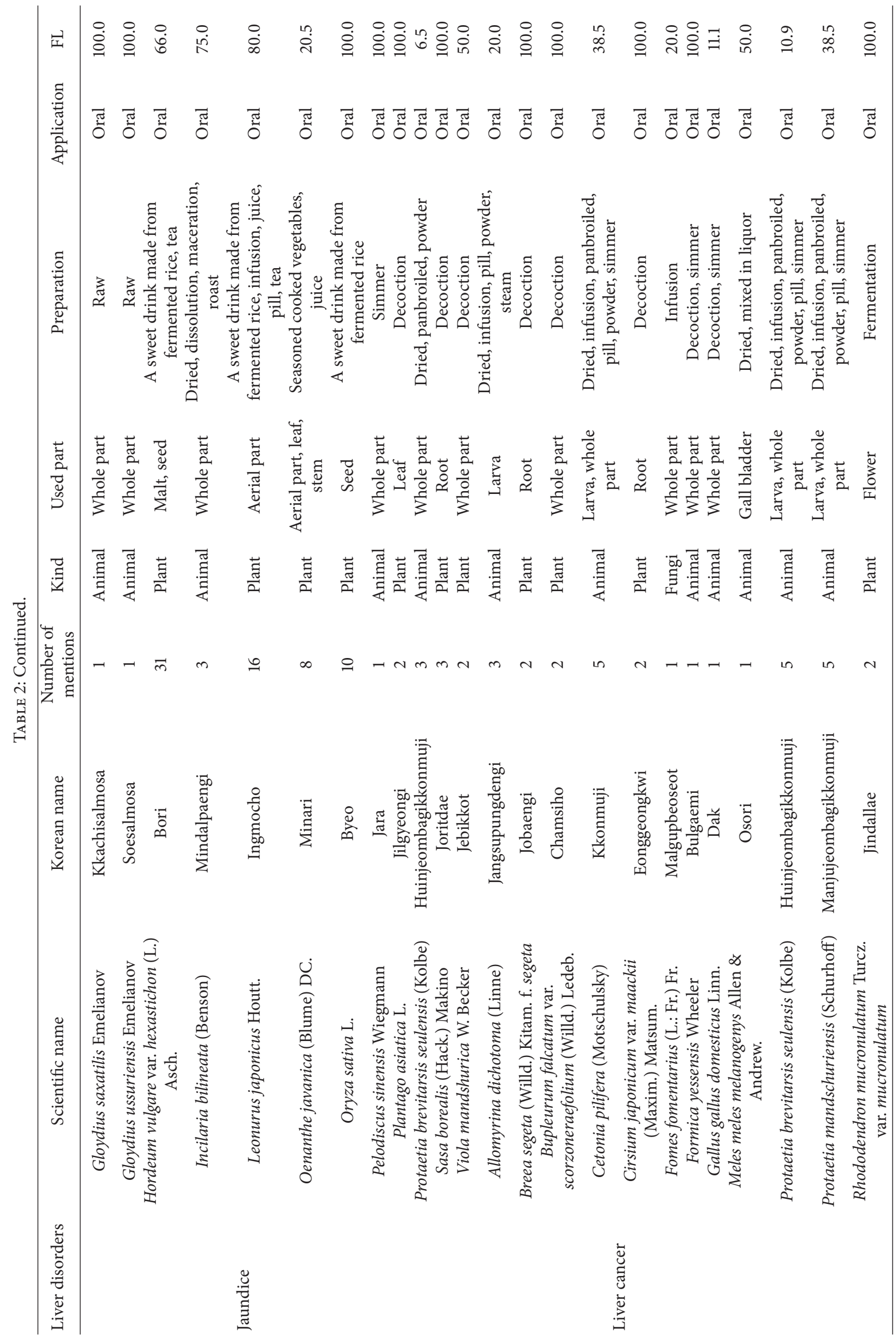




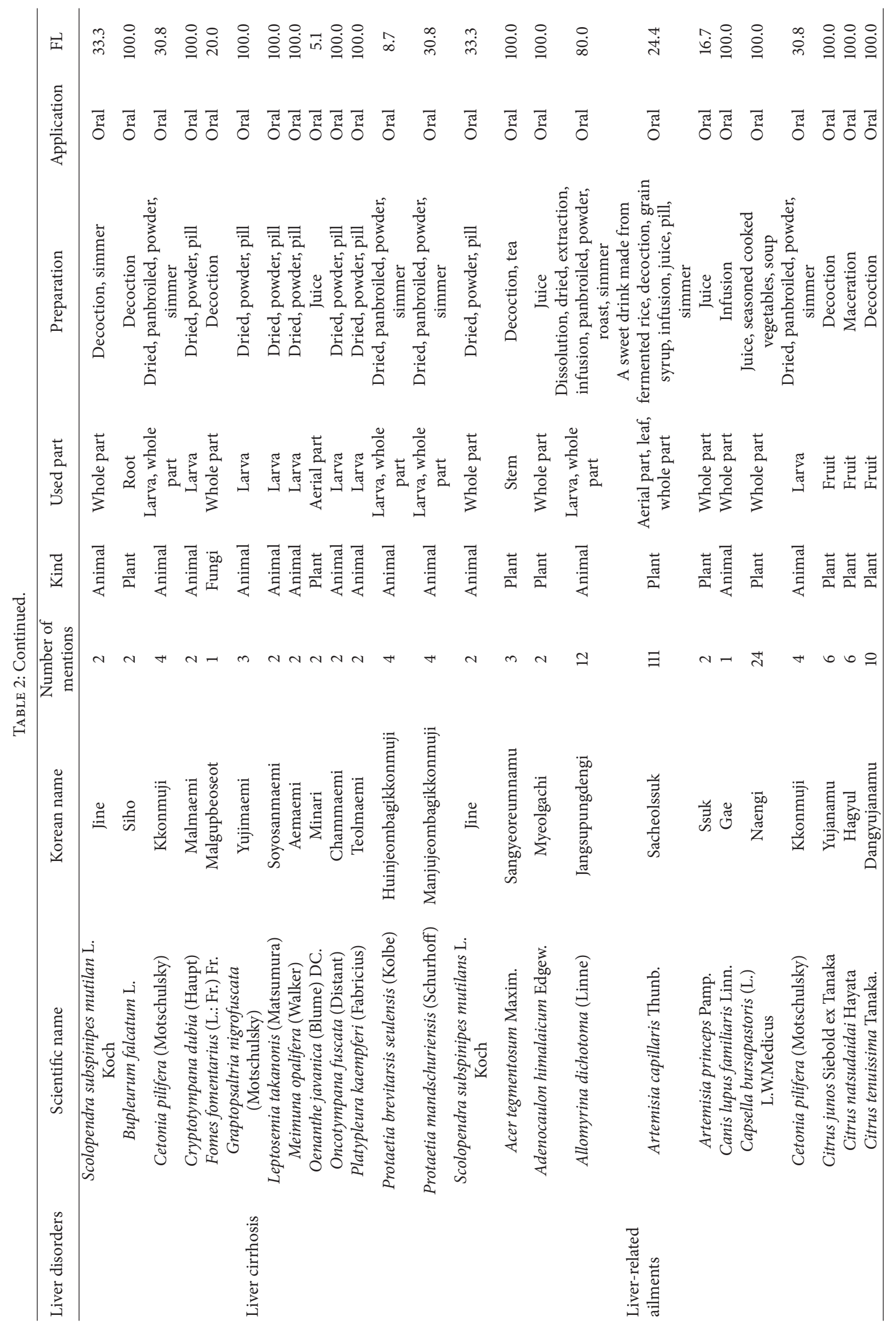




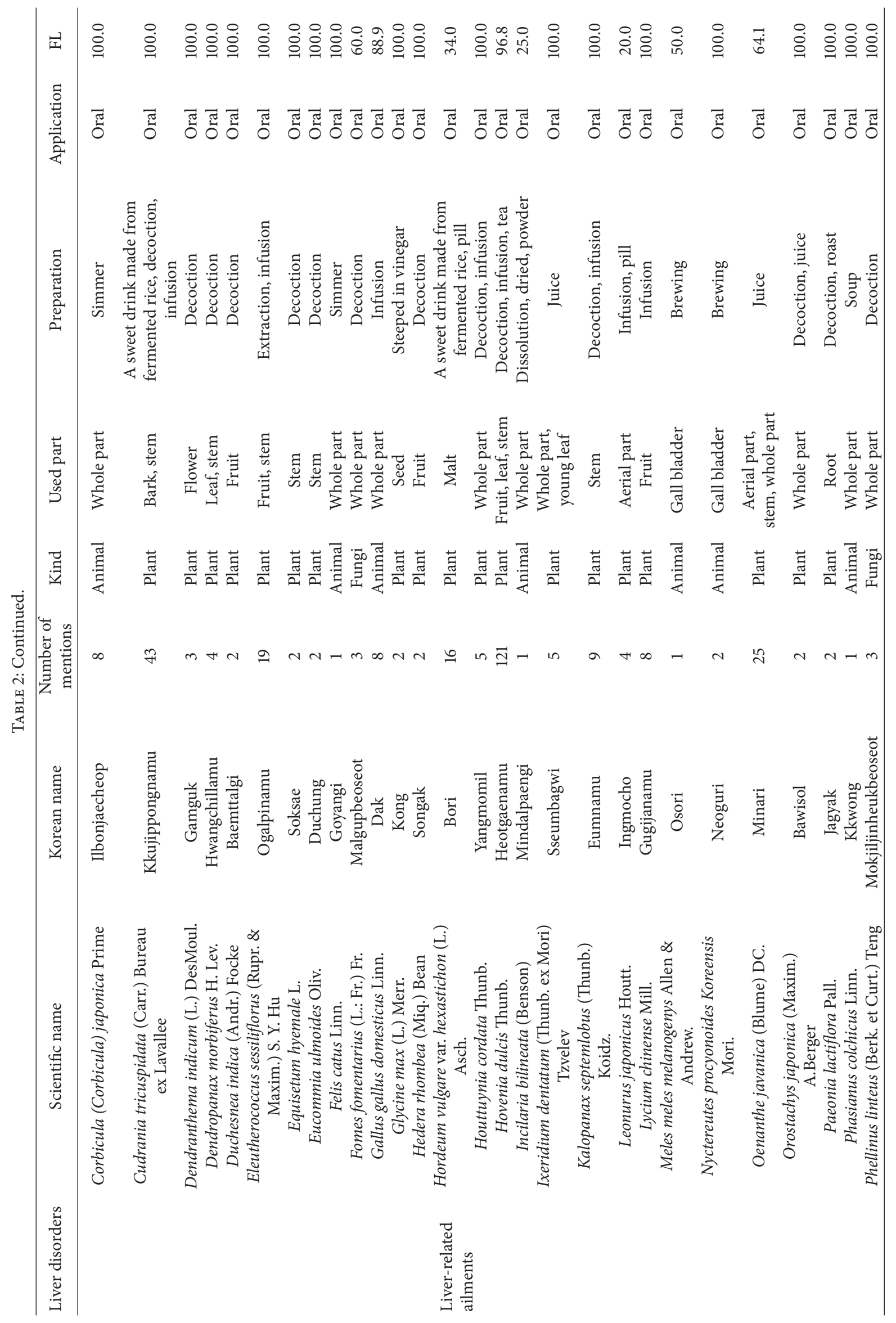




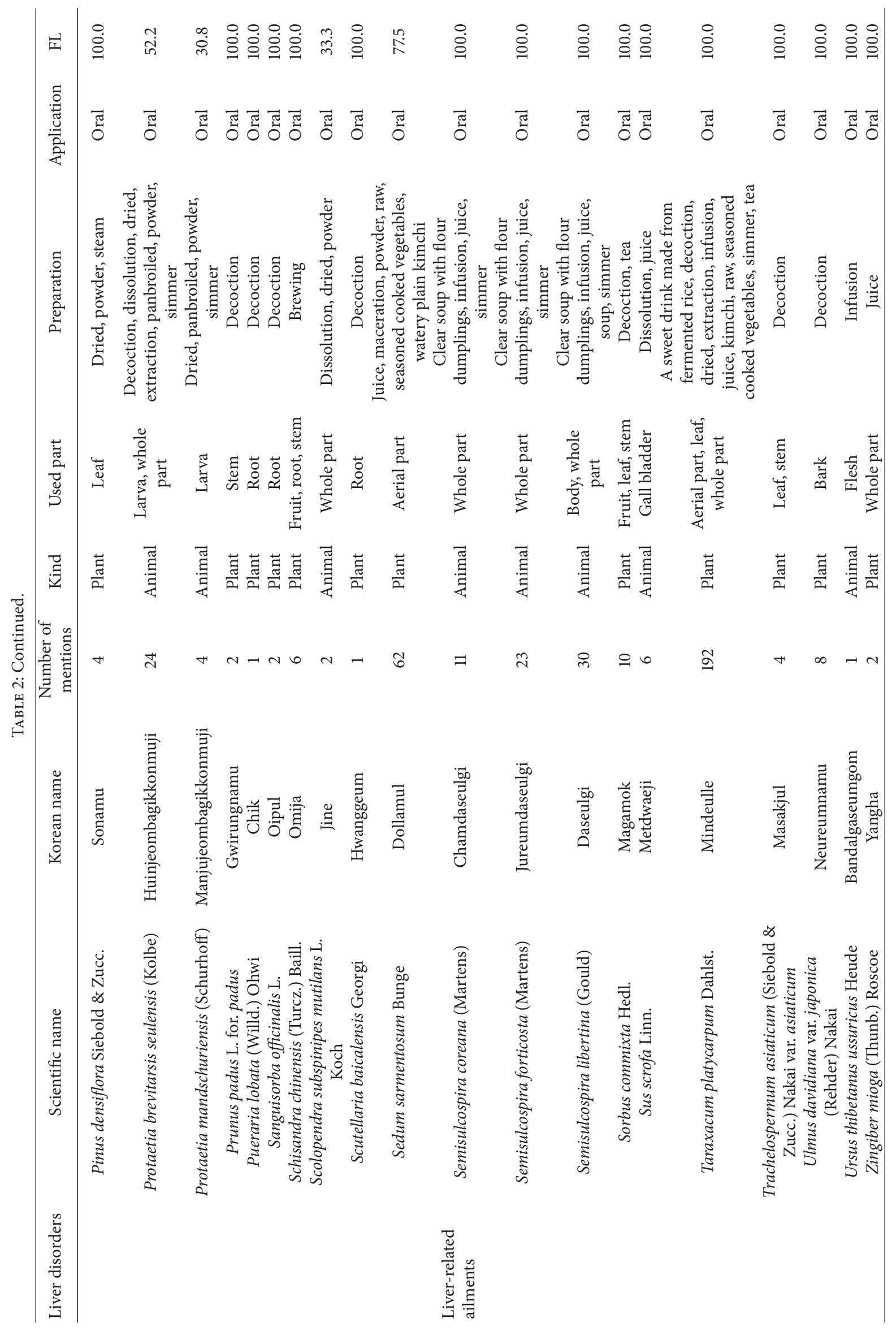


relative health issues related to liver conditions compared to other health concerns.

The 254 ethnomedicinal practices recorded from the communities were classified into 55 families, 85 genera, and 94 species that included plants, animals, and fungi (Table 2). Among these species, plants totaled 150 ethnomedicinal practices based on 31 families, 52 genera, and 57 species while animals included 99 ethnomedicinal practices based on 21 families, 30 genera, and 34 species. Fungi recorded five ethnomedicinal practices based on three families, three genera, and three species. These usage patterns were different from Korean traditional medicine, in which plants are used relatively much more than animals. Research confirms that the communities have focused on direct nutritional supplements from the traditional medicine rather than seek after an actual cure for their liver disorders.

This supposition was confirmed by the fact that liverrelated ailments and jaundice require a greater necessity for nutritional supplements than other diseases which are contained within many medicinal species and various ethnomedicinal practices.

Namely, the number of medicinal species and ethnomedicinal practices for liver-related ailments consisted of 59 species (62.7\% of the total species) and 143 ethnomedicinal practices (56.3\% of the total practices). Jaundice used 25 species (26.6\% of the total species) and 50 ethnomedicinal practices (19.7\% of the total practices).

Also, the number of informants who mentioned liverrelated ailments and cases of jaundice occupied 58\%, which totaled $32 \%$ of the whole, respectively (Table 2). As a result, the communities tended to use ethnomedicinal practices to care for their overall health instead of as a cure for a longterm condition.

For plants, 13 used parts were used in practice, while 9 used parts of animals and one used part of fungi were used in treatment. Preparations of the plants consisted of 24 kinds, with 19 preparations for animals and two preparations for fungi (Table 2). The usage recorded is similar to previous research for other diseases [7-9].

Among the medicinal species, the most often mentioned plants were Artemisia capillaris Thunb., Taraxacum platycarpum Dahlst., and Hovenia dulcis Thunb. (50.99\% mentioned), while the common animals were Protaetia brevitarsis seulensis (Kolbe), Semisulcospira libertina (Gould), and Semisulcospira forticosta (Martens) (6.90\% mentioned). The number of mentioned plants focused more on minor species than animal species. Through continued research, these species can certainly be developed into functional foods for particular liver disorders.

\subsection{Quantitative Analysis}

3.3.1. Informant Consensus Factor (ICF). The informant consensus factor ranges from 0 to 1 , where the increasing values indicate a higher rate of informant consensus among the illness category. The category with the highest degree of consensus from the informants was jaundice (0.95), followed by liver-related ailments and fatigue recovery (0.93), hepatitis (0.87), and a hangover (0.86). The lowest degree of consensus was for liver cancer and liver cirrhosis (0.61). These results inform that ethnomedicinal practices have been applied more often to minor health issues related to the liver.

More often, people suffering from serious liver disorders have been treated in the hospital using conventional medicine or Korean traditional medicine. However, ethnomedicinal practices have been used to cure jaundice, liver-related ailments, and fatigue recovery.

3.3.2. Fidelity Level (FL). The FL is useful for identifying the informants' most preferred species in use for treating certain liver disorders.

This information reveals that the informants had a tendency to rely on one specific species for treating one specific ailment rather than for several different ailments. The FL values in this study varied from $1.0 \%$ to $100 \%$.

Generally, a FL of $100 \%$ for a specific species indicates that all of the usereports mentioned the same species for a specific treatment [32]. This study determined 28 species of plants with a FL of $100 \%$, even without considering species that were mentioned above five times (Table 2). Diseases containing a higher number of species assessed to a FL of $100 \%$ were liver-related ailments (43 species) and cases of jaundice (16 species).

Special attention was given to important species $(N$, $N_{p}$ ) with a FL above $100 \%$, regarding the viewpoint of the number of times mentioned and the consensus level for the specific ailment, like Taraxacum platycarpum Dahlst. $(192,192)$, Cudrania tricuspidata (Carr.) Bureau ex Lavallee $(43,43)$, Semisulcospira libertina (Gould) $(30,30)$, Capsella bursapastoris (L.) L. W. Medicus (24, 24), and Semisulcospira forticosta (Martens) $(10,10)$ (Table 2). Through further clinical study, these species possess a much higher potential in being used in the development of new drugs for liver disorders.

\subsubsection{Internetwork between Liver Disorders and Medicinal} Species. INA has originally analyzed social phenomenon and trends through the internetwork of components [33]. We attempted to analyze the interrelationship between liver disorders and the medicinal species recorded in the communities.

Considering Figure 2, the people in the communities used only animals to care for fatigue recovery and liver cirrhosis, except for the use of Bupleurum falcatum L. (plant) and Fomes fomentarius (L.:Fr.) Fr. (fungus), while using plants and fungi as a cure for hepatitis and hangovers. Also, people used plants, animals, and fungi to treat jaundice, liver cancer, and other liver-related ailments.

Protaetia brevitarsis seulensis (Kolbe), used as a medicinal animal, was applied as treatment for five liver disorders, which included liver cancer, liver cirrhosis, jaundice, liverrelated ailments, and hepatitis. Oenanthe javanica (Blume) DC., used as a medicinal plant, was applied as treatment for the four liver disorders of liver cirrhosis, jaundice, liverrelated ailments, and hangovers. Protaetia mandschuriensis (Schurhoff) and Cetonia pilifera (Motschulsky) were used as medicinal animals and Fomes fomentarius (L.: Fr.) Fr. as a medicinal fungus in the treatment of three disorders, 


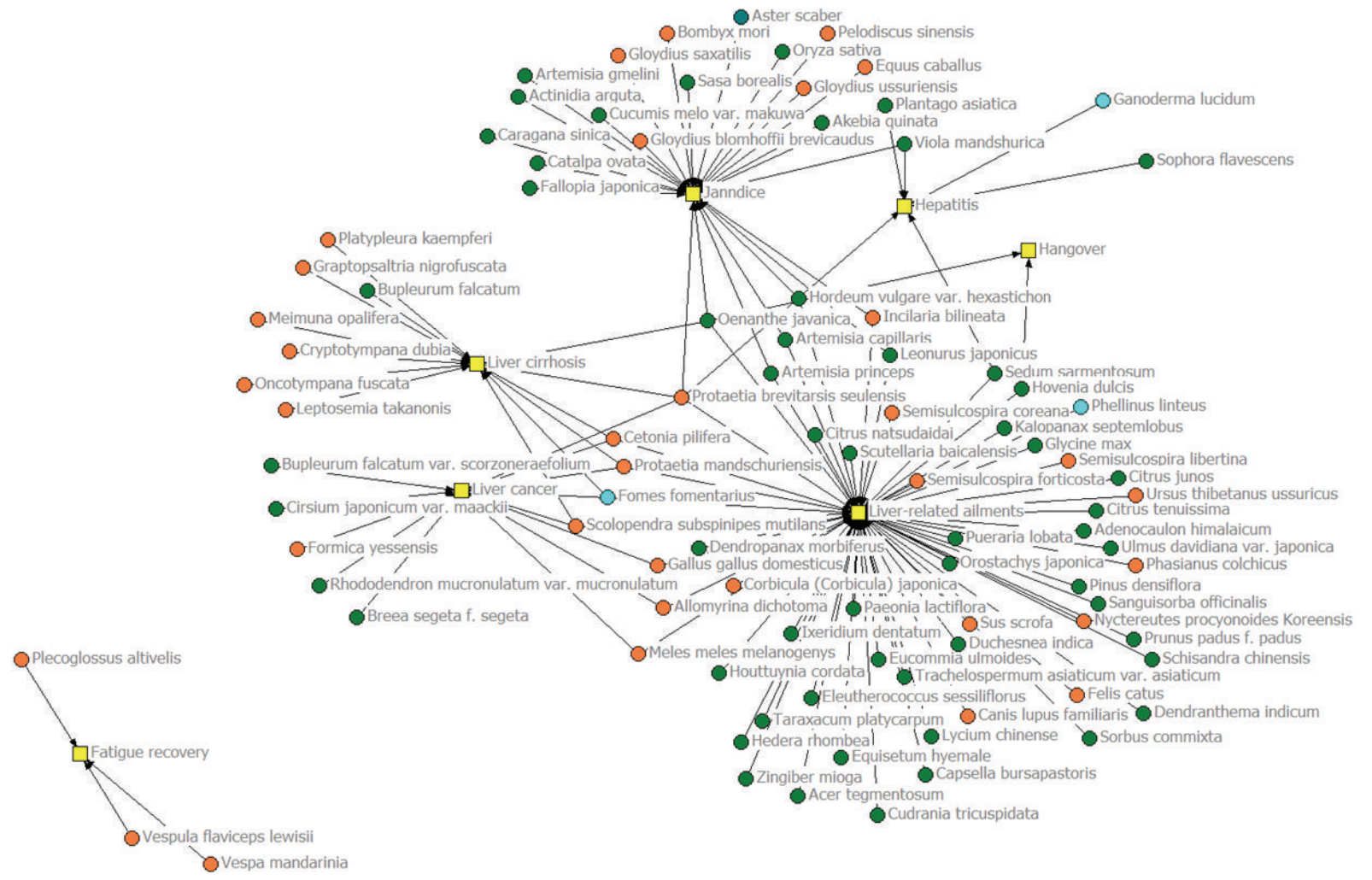

FIGURE 2: Internetwork analysis between medicinal species and diseases.

which included liver cancer, liver cirrhosis, and liver-related ailments. As further research is conducted, these species will certainly be developed as pharma foods used in treatment of liver disorders.

\section{Conclusion}

This research stands as the first study to record and analyze ethnomedicinal practices used as treatment for liver disorders within the communities in East Asia. After the 1950s, the National Health Care System legally admitted conventional medicine and Korean traditional medicine, which resulted in the near extinction of ethnomedicinal practice in Korea. Also, the fast westernization of local communities in Korea has accelerated the loss of ethnomedicinal practices.

From this research, recording 254 ethnomedicinal practices, as being used to treat seven liver disorders, was very inspiring. Particularly, the present usage of various bioorganisms displays evidence as to which ethnomedicinal practices are continuously transmitted in the communities. However, this present situation is not sustainable because the communities of these study areas consist of an aging society. It has become necessary for appropriate measures to be taken to conserve these ethnomedicinal practices.

Optimistically, the INA was first applied for the interpretation of ethnomedicinal knowledge of a community, although it has been strictly used until now for social science in the analysis of social trends and phenomena through the interrelationship of specific components.
The results of the INA application in this study provide various interpretations between liver disorders and medicinal species. Our research suggests an internetwork analysis as a new tool for various interpretations to ethnomedicinal knowledge within a local community. Through this study, we are confident that the useful value of INA has been proven and the three dimensional relationships of these components will extend beyond the existing understanding of ethnomedicinal knowledge within local communities around the world.

\section{Acknowledgments}

The authors are very grateful to all the informants for sharing their oral traditional knowledge during the fieldwork surveys. They acknowledge the English review of Professor Heldenbrand Brian at Jeonju University.

\section{References}

[1] T. Bubela and E. R. Gold, Genetic Resources and Traditional Knowledge, Edward Elgar, Northampton, Mass, USA, 2012.

[2] V. Reyes-García, "The relevance of traditional knowledge systems for ethnopharmacological research: theoretical and methodological contributions," Journal of Ethnobiology and Ethnomedicine, vol. 6, no. 32, pp. 1-32, 2010.

[3] R. Anderson, "The efficacy of ethnomedicine: research methods in trouble," Medical anthropology, vol. 13, no. 1-2, pp. 1-17, 1991.

[4] P. M. Unikrishnan and M. S. Suneetha, Biodiversity, Traditional Knowledge and Community Health: Strengthening Linkages, Xpress Pte, Singapore, 2012. 
[5] D. J. Kim, The Medicinal History of Korea, Tamgudang, Seoul, Korea, 1981.

[6] J. Sharma, S. Gairola, R. D. Gaur, and R. M. Painuli, "The treatment of jaundice with medicinal plants in indigenous communities of the Sub-Himalayan region of Uttarakhand, India," Journal of Ethnopharmacology, vol. 143, no. 1, pp. 262291, 2012.

[7] H. Kim and M.-J. Song, "Traditional plant-based therapies for respiratory diseases found in North Jeolla Province, Korea," Journal of Alternative and Complementary Medicine, vol. 18, no. 3, pp. 287-293, 2012.

[8] H. Kim and M.-J. Song, "Oral traditional knowledge for the treatment of digestive system diseases investigated in north jeolla province, Korea," Journal of Medicinal Plant Research, vol. 5, no. 24, pp. 5730-5740, 2011.

[9] H. Kim and M. J. Song, "Oral traditional plant-based therapeutic applications for pain relief recorded in North Jeolla province, Korea," Indian Journal of Traditional Knowledge. In press.

[10] Ministry of Security and Public Administration, 2013, http:// www.mospa.go.kr.

[11] Korea Meteorological Administration, 2013, http://www.kma .go.kr.

[12] H. Kim and M. J. Song, Benefit-Sharing and Industrialization for Traditional Knowledge of Biological Genetic Resources: Prevention of Nagoya Protocol, World science, Seoul, Korea, 2011.

[13] H. Kim and M.-J. Song, "Analysis and recordings of orally transmitted knowledge about medicinal plants in the southern mountainous region of Korea," Journal of Ethnopharmacology, vol. 134, no. 3, pp. 676-696, 2011.

[14] M.-J. Song and H. Kim, "Ethnomedicinal application of plants in the western plain region of North Jeolla Province in Korea," Journal of Ethnopharmacology, vol. 137, no. 1, pp. 167-175, 2011.

[15] G. J. Martin, Ethnobotany: A Conservation Manual, Champman \& Hall, London, UK, 1995.

[16] H. Kim and M. J. Song, Ethnobotany, World Science, Seoul, Korea, 2008.

[17] T. B. Lee, Illustrated Flora of Korea, Hyangmunsa, Seoul, Korea, 1979.

[18] J. Y. Lee, Coloured Korean Mushroom, vol. 1, Academy Publishing, Seoul, Korea, 1993.

[19] D. G. Ahn, Illustrated Book of Korean Medicinal Herbs, Kyohak, Seoul, Korea, 2002.

[20] Y. N. Lee, Flora of Korea, Kyohak, Seoul, Korea, 2002.

[21] J. H. Park, Korean Folk Medicine With Color Pictures, Shinil books, Seoul, Korea, 2005.

[22] "National Knowledge and Information System for Biological Species (NKISBS)," 2013, http://www.nature.go.kr.

[23] M. Heinrich, A. Ankli, B. Frei, C. Weimann, and O. Sticher, "Medicinal plants in Mexico: healers' consensus and cultural importance," Social Science and Medicine, vol. 47, no. 11, pp. 1859-1871, 1998.

[24] M. Heinrich, S. Edwards, D. E. Moerman, and M. Leonti, "Ethnopharmacological field studies: a critical assessment of their conceptual basis and methods," Journal of Ethnopharmacology, vol. 124, no. 1, pp. 1-17, 2009.

[25] M. N. Alexiades, Selected Guidelines for Ethnobotanical Research-a Field Manual, vol. 10 of Advances in Economic Botany, The New York Botanical Garden, Bronx, NY, USA, 1996.

[26] R. W. Douglas and U. Johansen, Network Analysis and Ethnographic Problems: Process Models of a Turkish Nomad Clan, Lexington Books, Idaho Falls, Idaho, USA, 2006.

[27] N. A. Christakis and J. H. Fowler, "The spread of obesity in a large social network over 32 years," The New England Journal of Medicine, vol. 357, no. 4, pp. 370-379, 2007.
[28] N. A. Christakis and J. H. Fowler, "Social contagion theory: examining dynamic social networks and human behavior," Statistics in Medicine, vol. 32, no. 4, pp. 556-577, 2013.

[29] N. A. Christakis and J. H. Fowler, "Rejoinder to commentaries on social contagion theory," Statistics in Medicine, vol. 32, no. 4, pp. 597-599, 2013.

[30] S. P. Borgatti, NetDraw Software for Network Visualization, Analytic Technologies, Lexington, KY, USA, 2002.

[31] S. P. Borgatti, M. G. Everett, and L. C. Freeman, Ucinet for Windows: Software for Social Network Analysis, Analytic Technologies, Harvard, Mass, USA, 2002.

[32] K. Srithi, H. Balslev, P. Wangpakapattanawong, P. Srisanga, and C. Trisonthi, "Medicinal plant knowledge and its erosion among the Mien (Yao) in northern Thailand," Journal of Ethnopharmacology, vol. 123, no. 2, pp. 335-342, 2009.

[33] Y. H. Kim, Social Inter-Network Analysis, Parkyongsa, Seoul, Korea, 2013. 


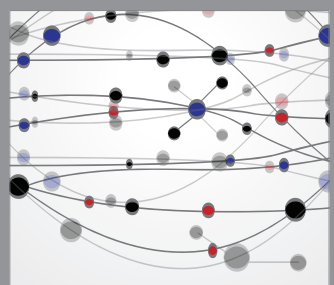

The Scientific World Journal
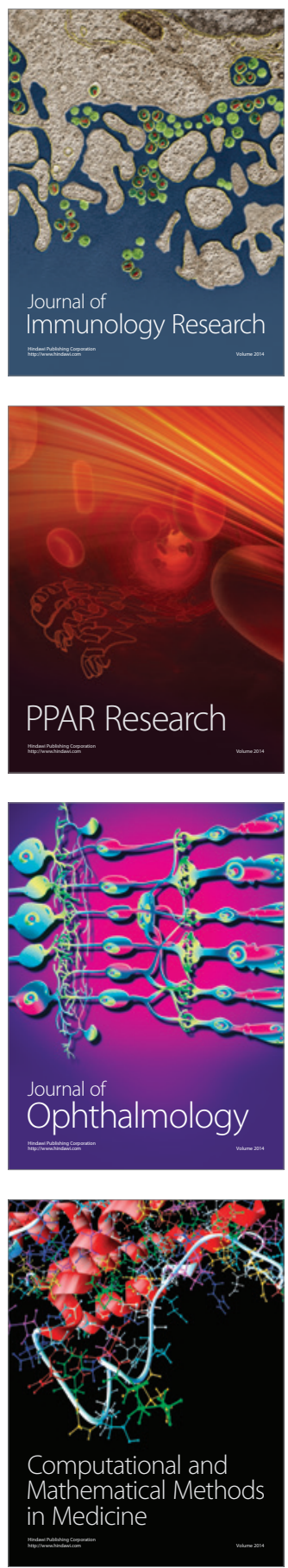

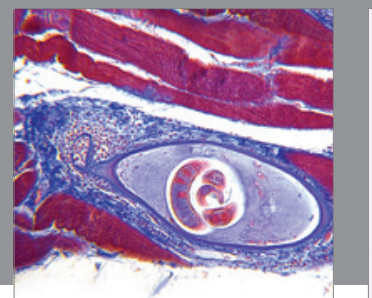

Gastroenterology

Research and Practice
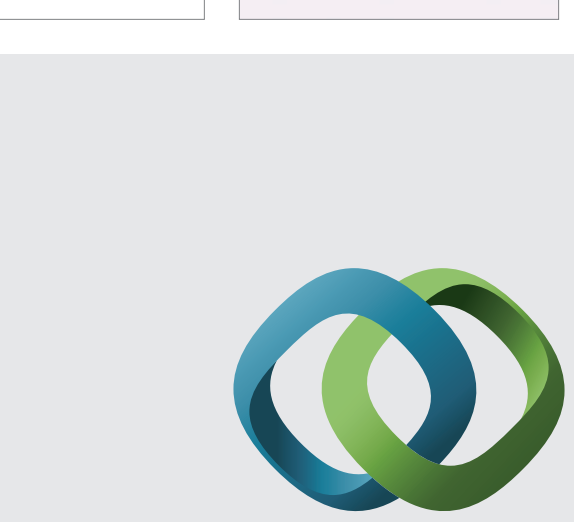

\section{Hindawi}

Submit your manuscripts at

http://www.hindawi.com
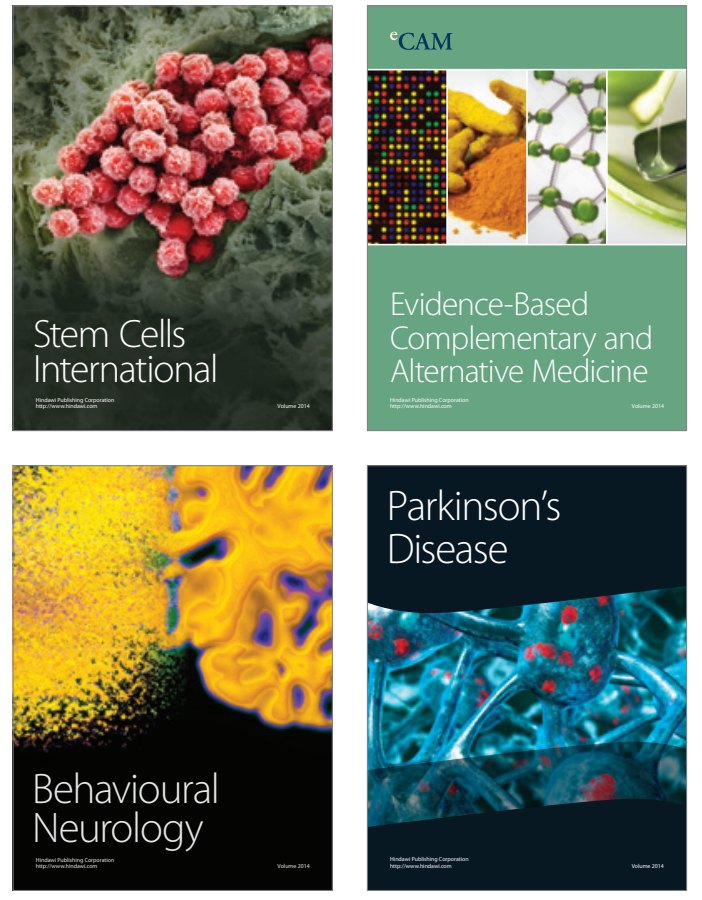
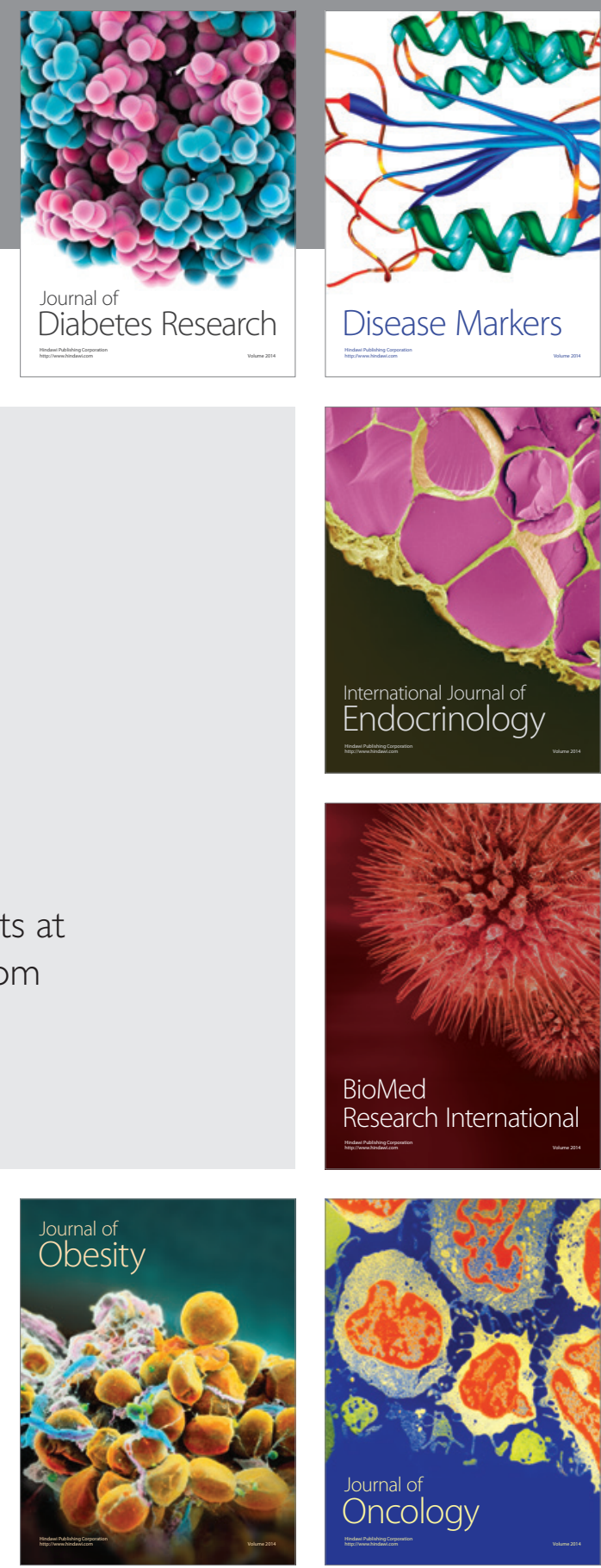

Disease Markers
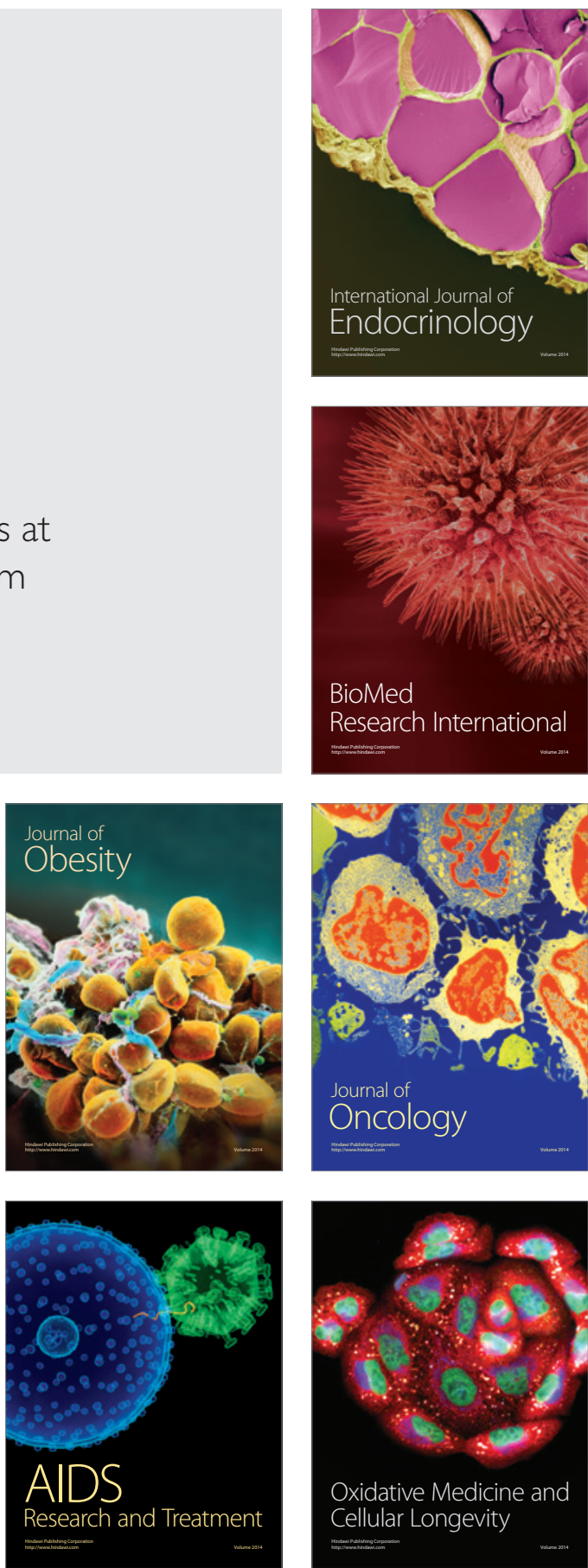